\title{
An Accessory Gluteus Maximus Muscle
}

\author{
By \\ *Serap Esin TUNCALI and Safiye CAVDAR \\ Marmara University Medical Faculty Anatomy Department \\ -Received for Publication, April 2, 1991 -
}

\begin{abstract}
Key Words: Accessory, Gluteus maximus, Variation
Summary: During the dissection of a $\mathbf{4 2}$ years old male cadaver, an accessory muscle contained in a seperate sheath of fascia was found at the inferior and anterior aspect of the right gluteus maximus muscle.
\end{abstract}

The gluteus maximus muscle is the largest, heaviest and most coarsely fibred muscle in the body which provides a thick quadrilateral pad over the ischial tuberosity. Structural changes in this muscle are critical to the evolutionary advance toward an upright stance during walking. Although its bilaminar nature ${ }^{1)}$ and its deep and superficial segmentation ${ }^{2)}$ are a part of our current knowledge, variations of the gluteus maximus muscle are not mentioned frequently in anatomy textbooks. These variations may have a clinical significance when the surgery of this region is under consideration.

\section{Materials and Methods}

During the routine dissection of 57 cadavers, an accessory muscle contained in its own fascial sheath was found at the inferior and anterior aspect of the right gluteus maximus muscle in a 42 years old male cadaver. The muscle was studied anatomically in detail and a specimen was examined under the light-microscope by the pathology department.

\section{Results}

The muscle was triangular shaped with an apex directed superio-medially and a long axis nearly parallel to the inferior fibres of the gluteus maximus (Figure $1,2)$. The total length of the muscle was $17 \mathrm{~cm}$. It passed inferomedial to the ischial tuberosity, partly inserting to the ramus of the ischium, and continued superomedially in the form of a $5 \mathrm{~cm}$ long, $0.7 \mathrm{~cm}$ wide tendon to blend with the sacrotuberous ligament. The muscular part extended laterally and inferiorly for 12 $\mathrm{cm}$. with a maximum width of $4.5 \mathrm{~cm}$, to blend with the inferior and internal fibres of the gluteus maximus muscle near its insertion. It was a flat muscle with an anterior surface facing the sciatic nerve and the common head of the biceps femoris and semitendinosis muscles. The posterior surface of the muscle faced the anterior surface of the gluteus maximus muscle. The maximum thickness was $0.5 \mathrm{~cm}$.

The gross and the light microscopic appearance of the muscle was normal.

Its blood supply was through a small branch from the first perforating branch of the femoral artery. Approaching it from its inferior aspect, the artery entered the muscle at its anterior surface near its inferolateral end at $15 \mathrm{~cm}$ distance from the coccygeal attachment. The same configuration of arterial supply to the inferolateral aspect of the gluteus maximus muscle was observed on the contralateral side.

\section{Discussion}

Phylogenetically, lower limb and pelvic girdle muscles are derived from the myotomes of L2-S3 levels ${ }^{3)}$. The gluteus maximus muscle is reported to arise from two muscle primordia; the fetal gluteus maximus muscle and the coccygeofemoralis muscle which later gradually fuse in prenatal life $\mathrm{e}^{4)}$. The pars sacroiliaca of the postnatal gluteus maximus muscle corresponds to the fetal gluteus maximus itself, and the pars coccygea represents the fetal coccygeofemoralis muscle ${ }^{4)}$.

The coccygeal attachment of this accessory muscle and the parallellity of its long axis with the inferior fibers of the gluteus maximus muscle gives the impression that it may be an unfused segment of the coccygeofemoralis muscle although the femoral attachment can not be traced.

The first perforating artery from the femoral artery 


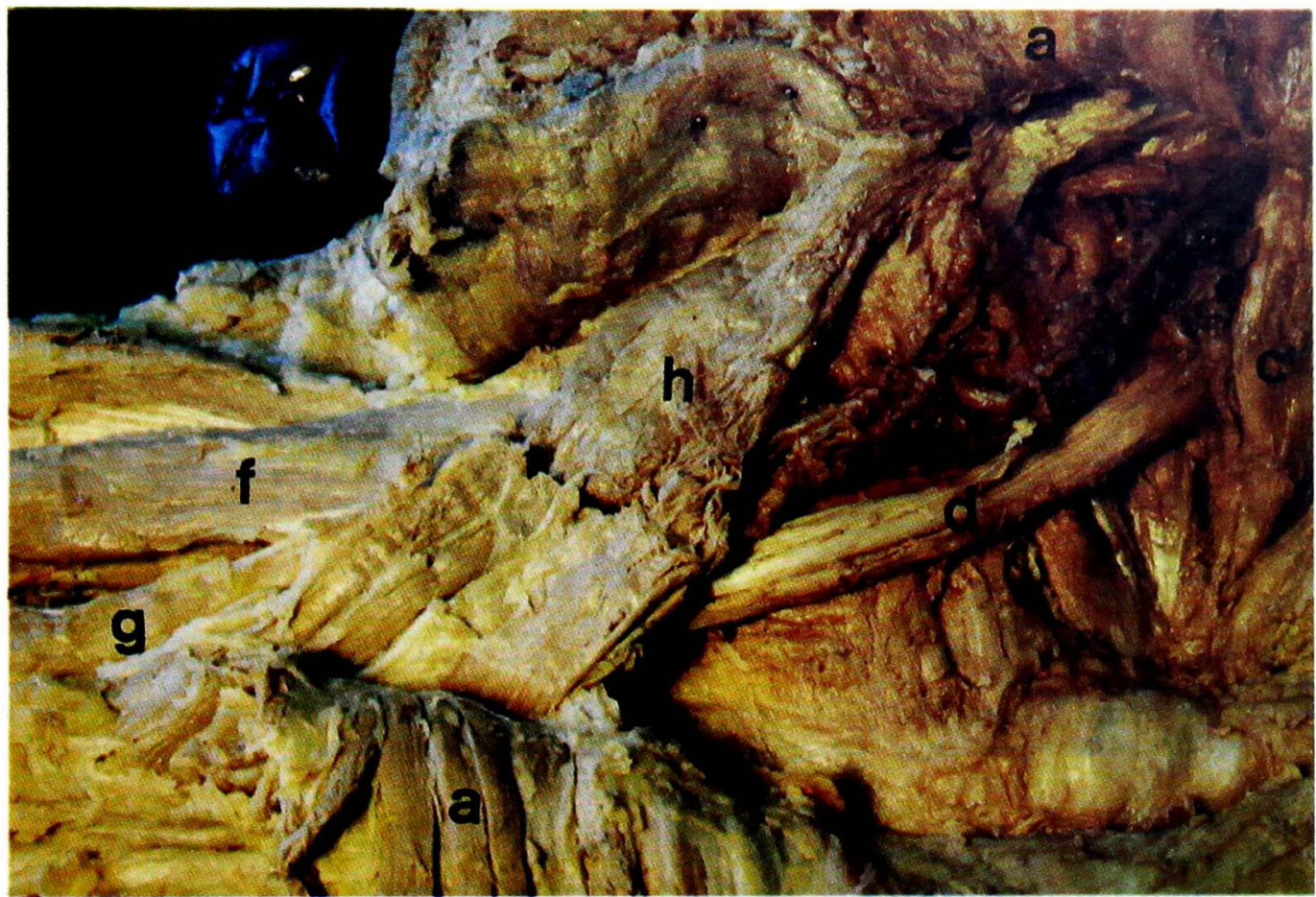

Fig. 1. The gross view of the right gluteal region; exposing the accessory gluteus maximus muscle.

is known to supply the distal part of the gluteus maximus muscle and the fascia, subcutaneous tissue and the skin over the lateral aspect of the thigh ${ }^{5)}$. The femoral and gluteal arterial anastomotic hemodynamics are affected by the relative involution of the gluteal system in late embryogenesis ${ }^{4}$. The rich vascular network of the gluteal and posterior thigh region provides for a large range of flaps for reconstructive surgery. The inferolateral entrance of the muscular branch of the first perforating artery to this accessory muscle and to the contralateral gluteus maximus muscle may have a clinical significance in designing musculocutaneous flaps.

\section{References}

1) Williams, Warwick. Gray's Anatomy. Churchill Livingstone, 36th ed. p.600-601, 1984.

2) Ramirez, O.M., Hurwitz, D.J., Futrell, J.W.: The expansive gluteus maximus flap. Plast. Reconstr. Surg., 74: 757-770, 1984.

3) Hamilton, W.J., Boyd, J.D., Mossman, H.W.: Human Embryology. Williams and Wilkins, 4th ed. p.553-559, 1972.

4) Tichy, M., Grim, M.: Morphogenesis of the human gluteus maximus muscle arising from two muscle primordia. Anat. Embryol. (Berl)., 173: 275-277, 1985.

5) Maruyama, Y., Ohnishi, K., Takeuchi, S.: The lateral thigh fascio-cutaneous flap in the repair of ischial and trochanteric defects. Br. J. Plast. Surg., 37: 103-107, 1984. 


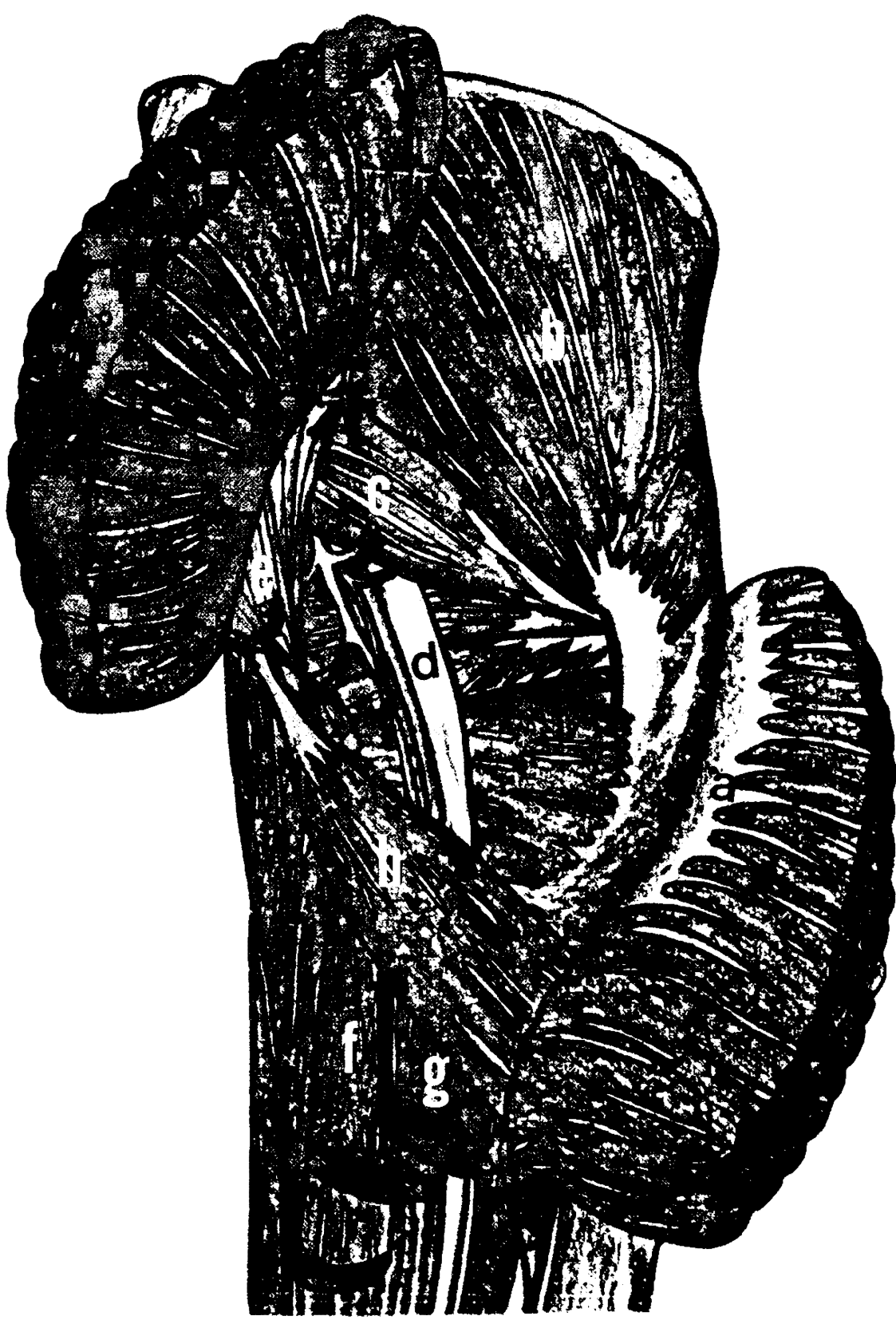

Fig. 2. A schematic drawing, showing the anatomical position of the accessory gluteus maximus muscle.
a: Gluteus maximus muscle
b: Gluteus medius muscle
c: Piriformis muscle
d: Sciatic nerve
e: Sacrotuberous ligament
f: Semitendinosis muscle
g: Biceps femoris muscle
h: Accessory gluteus maximus muscle 